\title{
Flotillin 1 is differentially expressed in human epithelial ovarian tumors
}

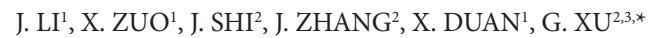 \\ ${ }^{1}$ Department of Obstetrics and Gynecology, Jinshan Hospital, Fudan University, Shanghai 201508, China; ${ }^{2}$ Center Laboratory, Jinshan Hospital, \\ Fudan University, Shanghai 201508, China; ${ }^{3}$ Department of Oncology, Shanghai Medical College, Fudan University, Shanghai 200032, China
}

${ }^{*}$ Correspondence: guoxiong.xu@fudan.edu.cn

Received July 14, 2017 / Accepted December 8, 2017

\begin{abstract}
Although Flotillin 1 (FLOT1) is highly expressed in various human cancers, its relationship with ovarian cancer (OC) remains unknown. This study determines FLOT1 expression in human ovarian tumors and examines its effect on OC cell proliferation. FLOT1 protein expression was assessed in a tissue microarray by immunohistochemical staining. We found that $81.48 \%$ malignant and $50 \%$ borderline tumors were FLOT1 protein-positive, whereas benign tumors and normal ovarian tissues were negative. The staining was strongest in serous malignant tumor and transitional cell carcinoma and weakest in mucinous tumor. Differentially expressed FLOT1 in freshly isolated serous tumors was confirmed by Western blot and we then evaluated FLOT1 expression association with OC patients' clinical characteristics. Histological typing established that FLOT1 protein expression was significantly associated with serous tumor $(\mathrm{P}<0.001)$, and that silencing FLOT1 by FLOT1-siRNA inhibited OVCAR-3 and SK-OV-3 cell proliferation and arrested the cell cycle at the S phase. FLOT1 inhibition increased cyclin E1 protein expression and over-expression partly rescued the FLOT1-shRNA-suppresed cell proliferation. Thus, we demonstrated that FLOT1 is highly expressed in ovarian cancer and its suppression decreases OC cell proliferation. This clearly suggests FLOT1's important role in ovarian tumor growth and provides a novel target for OC treatment.
\end{abstract}

Key words: cell proliferation, epithelial ovarian cancer, FLOT1, miRNA, therapeutic target, tumorigenesis

Ovarian cancer (OC) is the second most common gynecological malignancy and the eighth leading cause of global cancer death [1]. Further, 22,280 cases of OC with the highest mortality in all gynecological malignancies are diagnosed annually in America [2]. Over 70\% of these patients are diagnosed with advanced-stage disease, and their standard treatment is primary cyto-reductive surgery followed by platinum-based chemotherapy. Despite advances in surgery and chemotherapy, the 5-year OC survival has improved only slightly over the past 40 years and remains at $46 \%$ [3]. Therefore, early diagnosis and novel therapies are urgently required for OC effective treatment.

The flotillin proteins, including flotillin-1 (FLOT1) and flotillin-2 (FLOT2), are major protein components of lipid rafts [4] located not only in the plasma membrane but also in endosomes and in nuclear, Golgi and lysosomal compartments $[5,6]$. These molecules are involved in endocytosis, cell proliferation and adhesion and receptor mediated signal transduction [7-9]. FLOT1 mRNA and protein are overexpressed in various malignancies, including the following carcinomas with esophageal squamous cell [11], bladder transitional cell [12], hepatocellular [14] and renal cell carcinoma [16], and also in laryngeal [15], breast [13] and non-small lung cell cancers [10]. Over-expression of FLOT1 can promote cancer cell proliferation and tumorigenicity. Moreover, its down-regulation inhibits in vitro and in vivo cancer cell proliferation, invasion and metastasis while up-regulation promotes them [12, 17-19]. Further, high levels of FLOT1 expression positively correlate with the pathological stage and patient prognosis in several types of cancer $[10,14,20]$ but although FLOT1 appears involved in development and progression of a variety of cancers there have been no reports on the relationship between FLOT1 and OC.

BRCA1 is a susceptibility gene for ovarian and breast cancer [21], and people with inherited BRAC1 mutation have approximately $40-50 \%$ increased risk of developing OC [22]. Our previous proteomics study compared the immortalized BRCA1-mutated with immortalized wild-type ovarian surface epithelial cells and determined a series of differentially expressed proteins; including FLOT1, cystatin B 
(CSTB), $\beta$-2-microglobulin (B2M) and cytidine monophosphate kinase (CMPK). These proteins were higher in BRCA1mutated cells. Over-expression of CSTB, B2M and CMPK was further demonstrated in human epithelial ovarian cancer (EOC) and was mediated there by the TGF- $\beta$ transforming growth factor- $\beta$ signaling pathway [23-25]. Herein, we verified the differential expression of FLOT1 in normal ovarian tissue and benign, borderline and malignant tumors, and then elucidated FLOT1 knockdown effect on OC cell proliferation.

\section{Materials and methods}

Tissue microarray and immunohistochemical staining. A commercial tissue microarray (Xian Alena Biotechnology Co. Ltd., Xi'an, China) was used for the immunohistochemical analysis of 54 malignant tumors, 6 borderline tumors, 11 benign tumors and 18 normal ovaries. Clinical characteristics including age, histological tumor type, stage and differentiation grade were recorded, and tissue micro-array sections were then dewaxed and rehydrated before antigen retrieval. Tissues were incubated with a rabbit monoclonal anti-FLOT1 antibody at $4^{\circ} \mathrm{C}$ overnight (1:100 dilution, Cat\# ab133497, Abcam, Cambridge, UK) after blocking with normal goat serum for a minimum 10 minutes at room temperature (Fuzhou Maixin Biotech Co., Fuzhou, Fujian, China). After washing, tissues were incubated with biotinylated goat anti-rabbit secondary antibody at room temperature for 1 hour. (Fuzhou Maixin Biotech Co.). Signaling was detected by diaminobenzidine kit (DAB - Fuzhou Maixin Biotech Co.), and a micro-array of similar tissue without primary antibody formed the negative control.

Immunohistochemical staining analysis was performed by two independent pathologists without prior review of patient clinical data. The proportion of positive cells was scored as previously described [23]: $0=$ no positive cells; $1, \leq 25 \%$ positive cells; $2,26-50 \%$ positive cells; $3,51-75 \%$ positive cells and $4=>75 \%$ positive cells. Staining intensity was scored 0 for no staining; 1 = weak staining; 2 , moderate staining and 3 for strong staining. A final immunoreactivity score, or staining index (SI), was made by the sum of the positive proportion and staining intensity scores, with 0 as $\leq 2$ points; 1 as $3-4$ points; 2 as $5-6$ points; and 3 as 7 points. SI scores of 0 and 1 were FLOT1 negative; scores of 2 and 3 were FLOT1 positive.

Patients and tissue samples. Ovarian tissues used for Western blot analysis were collected from 12 patients with ovariectomy at Jinshan Hospital, Fudan University between December 2014 and June 2015. The Ethics Committee of Jinshan Hospital, Fudan University approved the study. Histological evaluation of tumors followed the current American Joint Committee on Cancer and the World Health Organization criteria of the tumor, node and metastasis (TNM) classification system The specimens included three malignant serous tumors, three benign tumors, three borderline tumors and three normal ovarian tissues. All patients with malignant tumors had been treated by cyto-reductive surgery without pre-operative therapy, and the remainder had ovariectomy or tumor resection.

Cell culture and small interfering RNA (siRNA) transfection. OVCAR-3 and SK-OV-3 human serous ovarian cancer cell lines were purchased from the American Type Culture Collection (Manassas, VA, USA). The OVCAR-3 cells were cultured in RPMI-1640 medium (Hyclone, Thermo Fisher Scientific Inc., Beijing, China) and SK-OV-3 cells in Dulbecco's Modified Eagle's Medium (DMEM, Hyclone), and both media were supplemented with $10 \%$ fetal bovine serum (Invitrogen, Carlsbad, CA, USA). The predesigned FLOT1siRNA sequences were sense 5'-GCCAAGGCACAGAGAGAUUTT-3' and antisense 5'-AAUCUCUCUGUGCCUUGGCTT-3'. The negative control-siRNA (NC-siRNA) sequences were sense 5'-UUCUCCGAACGUGUCACGUTT-3' and antisense 5'-ACGUGACACGUUCGGAGAATT-3'.

A 6-carboxyfluorescein-aminohexyphosphoramidite (FAM)labeled negative control siRNA (FAM-NC-siRNA) tested efficiency and optimized transfection conditions. The siRNA oligonucleotides were synthesized and purified by Shanghai Gene Pharma Co., Ltd (Shanghai, China). Cells were transfected by X-treme-GENE transfection reagent (X-TG, Roche, Penzberg, Germany) following manufacturer protocol. OVCAR-3 $\left(3 \times 10^{5} /\right.$ well $)$ and SK-OV-3 $\left(2 \times 10^{5} /\right.$ well $)$ cells were plated in six-well culture plates and transfected with $60 \mathrm{nM}$ siRNA. Wells with FAM-NC-siRNA were photographed by fluorescence microscopy (BX43, OLYMPUS, Tokyo, Japan) 6 hours after transfection in order to monitor transfection efficiency. Cells were then harvested 48 hours later for assay of transfection efficiency and further experimentation

Generation of FLOT1-shRNA-expressing cells and FLOT1-plasmid transfection. Human FLOT1 short hairpin RNA (shFLOT1) was constructed with double-strand oligonucleotides corresponding to the target sequence of 5'-GCCAAGGCACAGAGAGATT-3' and inserted into a pHY-LV-KD5.1 RNAi lentivirus vector containing green fluorescent protein (GFP) (hU6-MCS-CMV-ZsGreen 1-PGK-Puro, Hanyin Biotechnology Co., Ltd., Shanghai, China).

We then generated the FLOT1-shRNA-expressing cells and respective control cells. Cells were seeded in six-well plates overnight and infected with FLOT1-shRNA at concentration of 20 multiplicity of infection (MOI) with polybrene to a final concentration of $8 \mu \mathrm{g} / \mathrm{ml}$. Infection medium was replaced after 24 hours with fresh medium supplemented with $2 \mu \mathrm{g} / \mathrm{ml}$ puromycin to kill non-infected cells. The efficiency of FLOT1-shRNA lentiviral transduction was measured by fluorescence microscopy.

Human FLOT1 (NM_005803) plasmid (pFLOT1) (Cat\# EX-I0232-M51) and empty vector (EV) (Cat\# EX-I0232-M51) were purchased from GeneCopoeia Inc. (Rockville, MD, USA). FLOT1-shRNA-expressing cells were transfected with $2 \mu \mathrm{g}$ plasmid DNA by X-treme-GENE trans- 
fection reagent (Roche). FLOT1-shRNA-expressing cells at the fifth passage were used for the functional and rescue assays and FLOT1 protein over-expression was detected by Western blot.

Western blot analysis. Protein samples were collected from cells and tissues following standard protocols using sodium dodecyl sulfate (SDS) lysis buffer (Beyotime, Haimen, China) with $1 \%$ Phenylmethanesulfonyl fluoride (PMSF) plus phosphatase inhibitor (Jiangsu Keygen Biotech Corp., Ltd., Nanjing, Jiangsu, China). The protein samples were separated by $10 \%$ SDS-polyacrylamide gel electrophoresis (PAGE) and were transferred into a polyvinylidene fluoride membrane (PVDF - Millipore, Billerica, MA, USA). After blocking with $5 \%$ nonfat milk in Tris-buffered saline with Tween-20 (TBST) for 1 hour, the membranes were incubated at $4{ }^{\circ} \mathrm{C}$ overnight with either rabbit monoclonal anti-FLOT1 antibody (1:5000 dilution, Cat\# ab133497, Abcam) or mouse monoclonal anti-cyclin E1 antibody (1:1000 dilution, Cat\# 4128, Cell Signaling Technology, Inc., Danvers, MA, USA). The secondary antibodies were HRP-conjugated goat anti-rabbit IgG (1:5000 dilution, Cat\# KGAA35, KeyGEN BioTECH, Nanjing, Jiangsu, China) and goat anti-mouse IgG (1:5000 dilution, Cat\# KGAA37, KeyGEN BioTECH) kept at room temperature for 1 hour. Rabbit anti-GAPDH antibody (1:5000, Cat\# ab128915, Abcam) was used as loading control.

Cell proliferation assay. OVCAR-3 cells $\left(1 \times 10^{4} /\right.$ well $)$ and SK-OV-3 cells $\left(5 \times 10^{3} /\right.$ well $)$ in $100 \mu$ culture medium were added to 96-well culture plates. Cell proliferation was determined by adding $10 \mu \mathrm{l} /$ well WST-1 cell proliferation reagent following incubation for 24, 48 and 72 hours (Roche, Penzberg, Germany). The same volumes of culture medium and WST-1 were added to four blank wells to form the background control. Cells were then incubated in a humidified atmosphere at $37^{\circ} \mathrm{C}$ for 1 hour. After shaking thoroughly for 1 minute, the absorbance of the samples and the background controls were measured at $450 \mathrm{~nm}$ by microplate reader. This assay was repeated four times.

Cell cycle analysis. OVCAR-3 and SK-OV-3 cells were harvested 48 hours after transfection, washed with $1 \times$ phosphate buffered saline (PBS), fixed in cold $70 \%$ ethanol at $-20^{\circ} \mathrm{C}$ overnight and stained with $500 \mu \mathrm{l}$ propidium iodide (PI) solution for 30 minutes at room temperature in the dark. The number of cells at G1/G0, S, and G2/M phase of the cell cycle was determined by flow cytometry (Becton Dickinson Beckman Coulter, Inc., Brea, CA, USA) and data was analyzed in Multicycle for Windows 7 (San Diego, CA, USA).

Statistical analysis. Statistical analysis was performed by SPSS Statistics 17.0 for Windows (SPSS, Chicago, IL, USA). Fisher's exact test compared FLOT1 positivity differences in multiple groups and association between FLOT1 expression and clinical-pathological characteristics. The Wilcoxon rank-sum test compared immunostaining scores and the student's t-test compared two independent group mean values. One-way ANOVA (analysis of variance) with post-hoc test compared multiple group comparison, and results were reported as means \pm the standard error of mean from at least three independent experiments at $\mathrm{p}<0.05$ statistical significance.

\section{Results}

FLOT1 is differentially expressed in ovarian tumors. Positive staining of FLOT1 protein by immunohistochemistry was identified by brown coloring. FLOT1 protein expression was negative in serous benign tumor, moderate in borderline tumor and strong in malignant tumor (Figure 1A); with very low or no FLOT1 protein expression detected in mucinous tumor (Figure 1B). The highest staining intensity in malignant tumor was determined in serous and transitional cell malignant tumors (Figure 1A and 1C), moderate intensity was observed in endometrioid malignant tumor (Figure 1D), normal ovary tissues had no detectable FLOT1 protein level (Figure 1E) and serous malignant tumor without primary antibody was the negative control (Figure 1F). FLOT1 positivity was seen in $81.48 \%(44 / 54)$ of malignant tumor, $50 \%(3 / 6)$ of borderline tumor, $9.09 \%$ (1/11) of benign tumor and in $0 \%$ of $(0 / 18)$ of normal ovarian tissue. The percentage of FLOT1-positive cells was significantly higher in malignant tumor than in benign tumor and normal tissue (both $\mathrm{p}<0.001)$ and higher positivity rate was noted in borderline tumor than in normal tissue $(\mathrm{p}=0.01)$. No difference was established in positivity rates between normal tissue and benign tumor $(\mathrm{p}=0.379)$, benign and borderline tumors $(\mathrm{p}=0.099)$ or borderline and malignant tumors $(\mathrm{p}=0.109)$. Analysis of the FLOT1 protein expression immunoreactivity score in the various types of ovarian tissue yielded similar results (Table 1 ).

FLOT1 protein expression was further confirmed in 12 ovarian tissues by Western blot analysis. This included normal ovarian tissues, serous benign, borderline and malignant tumors ( $n=3$ per group); where FLOT1 protein expression was high in malignant and borderline tumors and low in benign tumors and normal ovarian tissues (Figure 2A). FLOT1 expression difference between malignant and benign tumors was significant at $\mathrm{p}<0.05$ and non-significant between

Table 1. Immunoreactivity score of FLOT1 protein expression in ovarian tissue.

\begin{tabular}{lcc}
\hline Comparison & Z score & p-value \\
\hline Normal vs. Benign & -3.429 & 0.001 \\
Normal vs. Borderline & -4.226 & $<0.001$ \\
Normal vs. Malignant & -6189 & $<0.001$ \\
Benign vs. Borderline & -1.708 & 0.094 \\
Benign vs. Malignant & -3.928 & $<0.001$ \\
Borderline vs. Malignant & -1.428 & 0.167 \\
\hline
\end{tabular}

FLOT1 immunoreactivity scores between two types of ovarian tissues were compared by the Wilcoxon rank-sum test. Normal, normal ovarian tissue; Benign, ovarian benign tumor; Borderline, ovarian borderline tumor; Malignant, ovarian malignant tumor. 

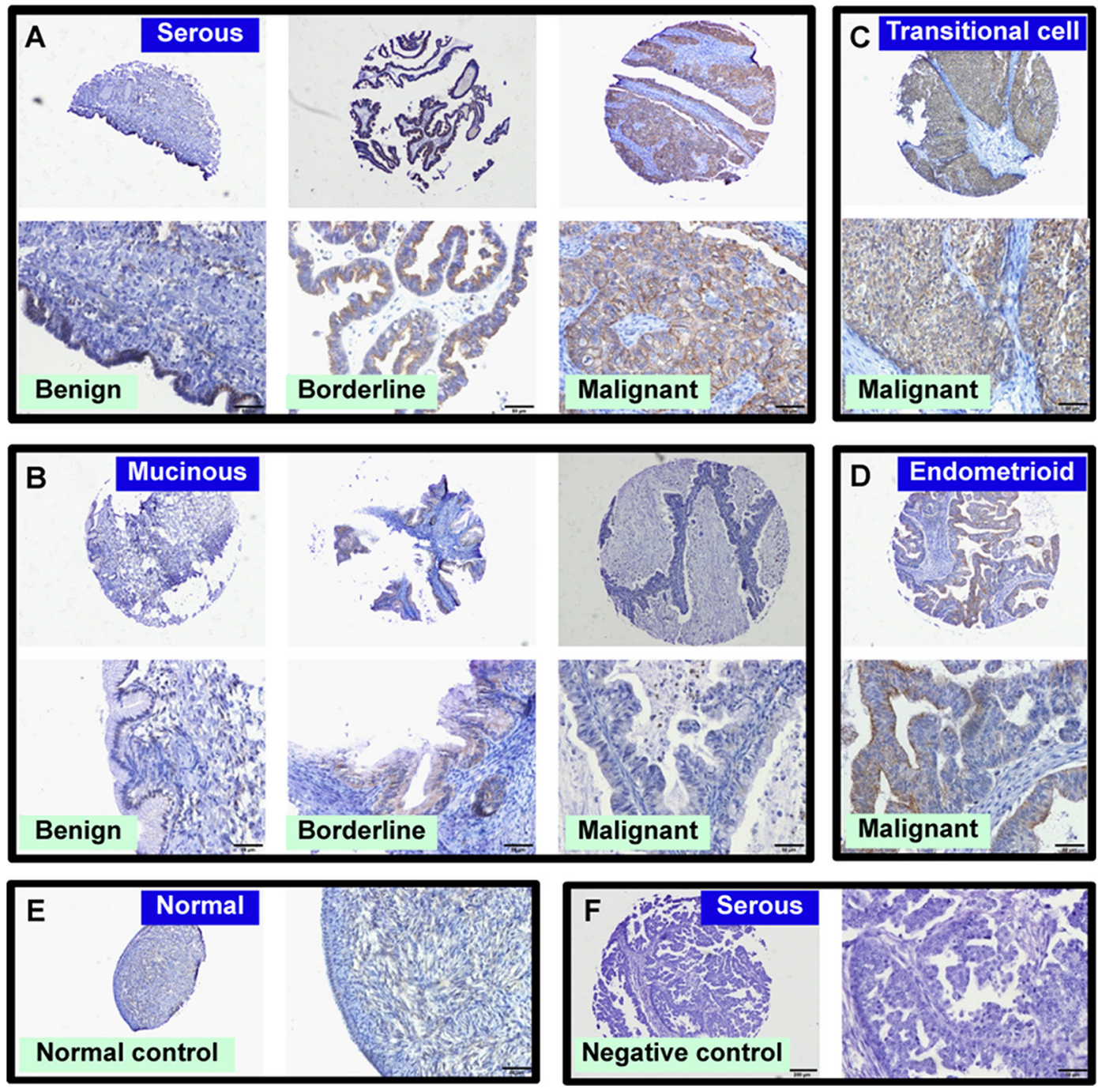

Figure 1. Immunohistochemical staining of FLOT1 protein in human ovarian tissues. Representative images of FLOT1 expression in A) serous benign, borderline, and malignant tumors, B) mucinous benign, borderline, and malignant tumors, C) transitional cell tumors, D) endometrioid, E) normal ovarian tissue, and F) negative control tissues. Positive staining is shown as brown coloring in epithelial cells. Original magnification $\times 400$. Scale bar, $50 \mu \mathrm{m}$.

malignant and borderline tumors (Figure 2B). Western blot and immunohistochemical staining results were consistent.

FLOT1 protein expression is associated with clinicalpathological features of patients with ovarian serous tumor. After immunochemistry staining in a tissue microarray, 89 samples remained on a glass slide and 11 samples were lost. Statistical analysis showed no correlation of FLOT1 protein expression with age, differentiation, clinical stage or lymph node metastasis (Table 2). Analysis of histological type detected that FLOT1 protein expression was significantly associated with serous tumor $(\mathrm{p}<0.001)$ and the positivity rate was higher in serous malignant tumor than in benign and borderline tumors. Further, positivity rates differed between the multiple malignant tumor types $(\mathrm{p}<0.001)$ : $91.89 \%(34 / 47)$ in serous, $0 \%(0 / 3)$ in mucinous, $60 \%(6 / 10)$ in endometrioid and $100 \%(4 / 4)$ in transitional tumors.
FLOT1-siRNA inhibits OVCAR-3 and SK-OV-3 cell proliferation. Transfection efficiency was estimated by FAM-NC-siRNA transfection of OVCAR-3 and SK-OV-3 cells. Over $80 \%$ of OVCAR-3 cells were positive after 6 hours transfection, with green coloring observed under fluorescence microscopy (Figure 3A). Results in SKOV3 cells were similar to OVCRA-3 cells (data not shown) and Western blotting indicated that FLOT1 protein expression significantly decreased after 48-hour transfection in FLOT1-siRNA transfected cells; thus confirming satisfactory transfection efficiency (Figures 3B-3E). FLOT1 silencing effect on OVCAR-3 and SK-OV-3 cell proliferation was determined by the WST-1 assay 48 hours after transfection. Figures $3 \mathrm{~F}$ and $G$ reveal that cell proliferation was inhibited in FLOT1siRNA transfected cells compared to the Blank, Reagent and NC-siRNA controls $(\mathrm{p}<0.05)$. 

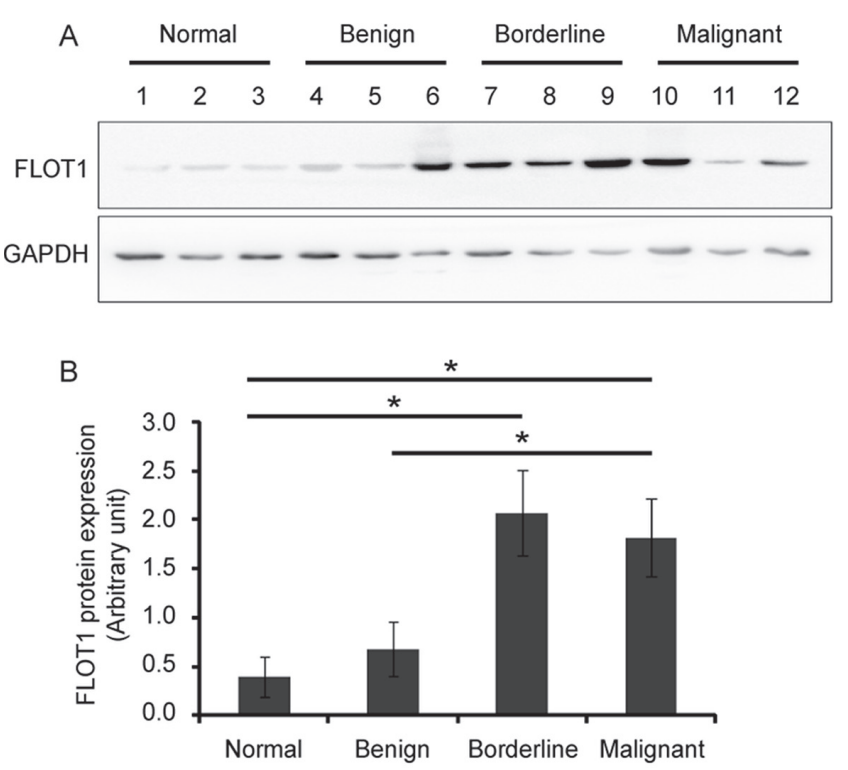

Figure 2. FLOT1 protein expression in serous tumors and normal ovarian tissue. A) Western blot analysis in normal ovarian tissue (case \#1-3) and ovarian serous benign (case \#4-6), borderline (case \#7-9), and malignant (case \#10-12) tumors using specific antibody against FLOT1. GAPDH was used as a loading control. B) Semi-quantitative analysis after densitometry on the gels of $(\mathrm{A}) .^{*}, \mathrm{p}<0.05$.

Overexpression of FLOT1 rescues the FLOT1-shRNAsuppresed cell proliferation. FLOT1-shRNA confirmed that FLOT1 knockdown (shFLOT1) inhibition of OVCAR-3 (Figure 4A) and SK-OV-3 cell proliferation began at 24 hours post-infection compared to the negative control (shNC - Figure 4B). FLOT1 over-expression by FLOT1 plasmid DAN (pFLOT1) significantly increased OVCAR-3 and SK-OV-3 (Figures 4 C, D) cell proliferation $(\mathrm{p}<0.05)$. We then performed rescue assay.

The FLOT1-shRNA-expressing cells (shFLOT1) derived from OVCAR-3 and SK-OV-3 (Figures 4E, F) were transfected with pFLOT1 for 72 hours and we again observed cell proliferation inhibition in shFLOT1-expressing cells. This was partly rescued after FLOT1 over-expression $(\mathrm{p}<0.05)$.

Inhibition of FLOT1 increased cyclin E1 protein expression. Stable shFLOT1- and shNC-expressing cell lines were generated in SK-OV-3 cells after infecting cells with FLOT1shRNA and NC-shRNA, respectively, and we confirmed that FLOT1 protein expression was significantly lower in shFLOT1-expresssing cells than in shNC control cells $(\mathrm{p}<0.01$; Figure $5 \mathrm{~A})$. FLOT1 protein expression in SK-OV-3 and shFLOT1-expressing cells was significantly increased after FLOT1-plasmid (pFLOT1) transfection compared to the empty vector control (EV) $(\mathrm{p}<0.05$; Figures $5 \mathrm{~B}, \mathrm{C})$. Tests for FLOT1 effect on cyclin E1 proliferation marker expression determined that it was significantly increased in shFLOT1-expressing cells ( $\mathrm{p}<0.05$; Figure 5D). Over-expression of FLOT1 decreased this expression $(\mathrm{p}<0.05$; Figure $5 \mathrm{E})$
Table 2. Association between FLOT1 protein expression and clinicopathological characteristics of patients with ovarian epithelial tumor.

\begin{tabular}{|c|c|c|c|c|}
\hline \multirow{2}{*}{$\begin{array}{l}\text { Clinicopathological } \\
\text { features }\end{array}$} & \multirow{2}{*}{$\mathbf{n}$} & \multicolumn{2}{|c|}{ FLOT1 expression } & \multirow{2}{*}{ p-value } \\
\hline & & Positive (\%) & Negative (\%) & \\
\hline Age at diagnosis & & & & $0.660^{a}$ \\
\hline$\leq 45$ & 32 & $16(50.00)$ & $16(50.00)$ & \\
\hline$>45$ & 57 & $32(56.14)$ & $25(43.86)$ & \\
\hline \multicolumn{5}{|l|}{ Histological type } \\
\hline Serous tumour & & & & $<0.001^{b}$ \\
\hline Benign & 3 & $0(00.00)$ & $3(100.00)$ & \\
\hline Borderline & 4 & $3(75.00)$ & $1(25.00)$ & \\
\hline Malignant & 37 & $34(91.89)$ & $3(8.11)$ & \\
\hline Mucinous tumour & & & & $1.000^{b}$ \\
\hline Benign & 8 & $1(12.50)$ & $7(87.50)$ & \\
\hline Borderline & 2 & $0(00.00)$ & $2(100.00)$ & \\
\hline Malignant & 3 & $0(00.00)$ & $3(100.00)$ & \\
\hline Malignant tumor & & & & $<0.001^{b}$ \\
\hline Serous & 37 & 34 (91.89) & $3(8.11)$ & \\
\hline Mucinous & 3 & $0(00.00)$ & $3(100.00)$ & \\
\hline Transitional cell & 4 & $4(100.00)$ & $0(00.00)$ & \\
\hline Endometrioid & 10 & $6(60.00)$ & $4(40.00)$ & \\
\hline Differentiation & & & & $0.160^{b}$ \\
\hline Well & 29 & $26(89.66)$ & $3(10.34)$ & \\
\hline Moderate & 15 & $10(66.67)$ & $5(33.33)$ & \\
\hline Poor & 10 & $8(80.00)$ & $2(20.00)$ & \\
\hline FIGO stage & & & & $0.609^{b}$ \\
\hline I & 24 & $18(75.00)$ & $6(25.00)$ & \\
\hline II & 11 & $10(90.91)$ & $1(9.09)$ & \\
\hline III & 9 & $7(77.78)$ & $2(22.22)$ & \\
\hline LN metastasis & & & & $1.000^{a}$ \\
\hline Yes & 9 & $7(77.78)$ & $2(22.22)$ & \\
\hline No & 35 & $28(80.00)$ & $7(20.00)$ & \\
\hline
\end{tabular}

The expression of FLOT1 protein was detected by immunohistochemistry using tissue microarray. The $\chi^{2}$ test (a). compared FLOT protein expression associated with age and metastasis.Fisher's exact test (b). determined multiple comparisons of FLOT1 protein expression associated with histological types, clinical stages and pathological differentiation.

$(\mathrm{n}=$ number of cases; Positive, positive expression; Negative, negative expression; LN, lymph node and FIGO = International Federation of Gynaecological Oncologists. Normal ovarian tissue $(n=18)$ with negated FLOT1 staining served as control.

and it was also decreased in shFLLOT1-expressing cells after pFLOT1 transfection $(\mathrm{p}<0.05$; Figure $5 \mathrm{~F})$.

FLOT1-siRNA arrests SK-OV-3 and OVCAR-3 cell cycles. The OVCAR-3 and SK-OV-3 cell cycles were assayed by flow cytometry to investigate suppression mechanisms in cell proliferation associated with FLOT1 knockdown. The G0/G1-phase population in FLOT1 silencing cells decreased and the S-phase population increased compared to control group cell cycles $(p<0.05$; Figure 6); FLOT1 knockdown arrested the cell cycle in the $S$ phase but did not effect G2/M. The overall results suggest that FLOT1 suppression inhibited ovarian cancer cell growth by blocking cell cycle progression. 

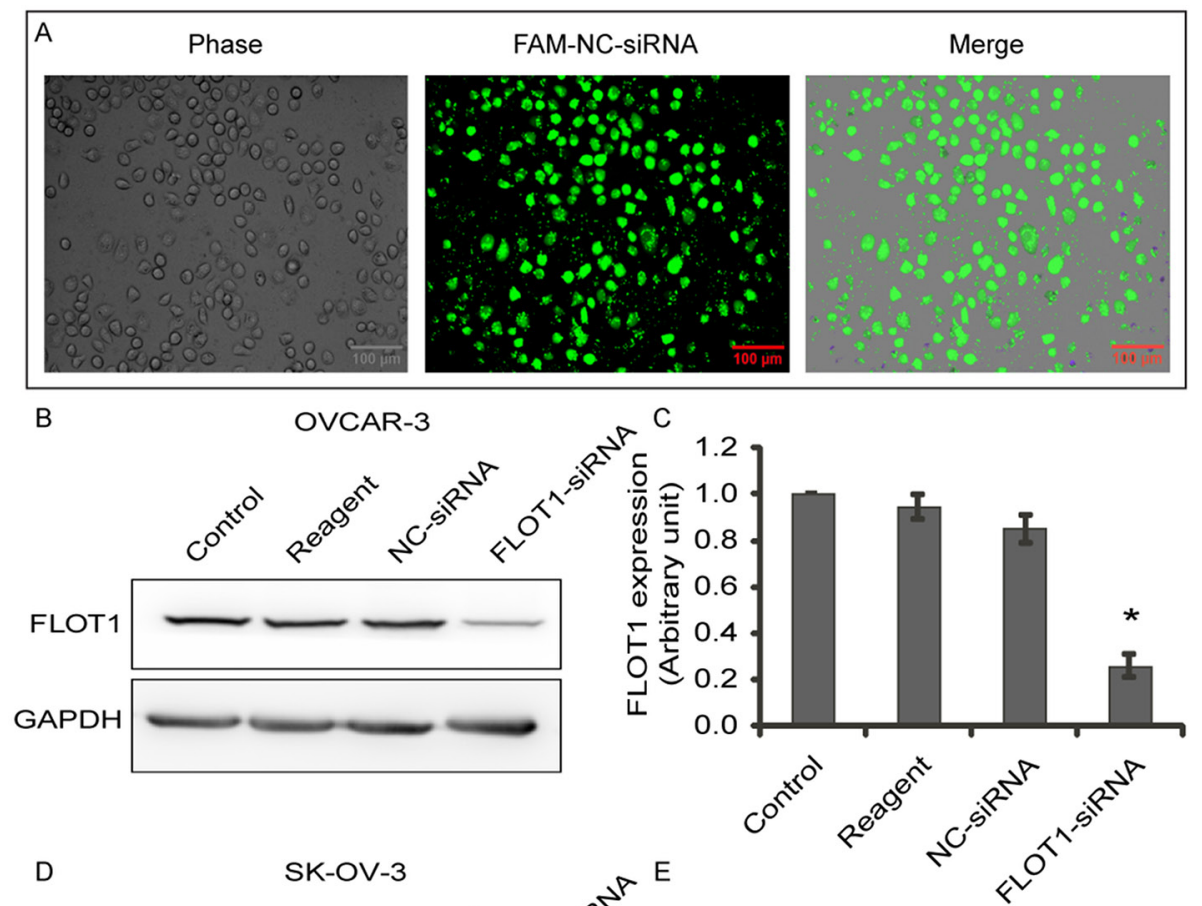

Figure 3. Efficiency of FLOT1-siRNA transfection and cell proliferation. A) OVCAR-3 cells were transfected with NCFAM siRNA with the final siRNA concentration of $60 \mathrm{nM}$. Cells in the same visual field were observed and photographed 6 hours later by light microscopy (left picture) and fluorescence microscopy (middle picture). The right picture shows a merged image of phase and FAM fluorescence. B) Western blot analysis of FLOT1 protein expression in OVCAR-3 cells after FLOT1siRNA transfection. C) Histogram shows the semi-quantitative analysis after densitometry on the gels of $(B)(n=4)$. D) Western blot analysis of FLOT1 protein expression in SK-OV-3 cells after FLOT1-siRNA transfection. E) Histogram shows the semiquantitative analysis after densitometry on the gels of $(D)(n=4)$. Cell proliferation was decreased after FLOT1-siRNA transfection in OVCAR-3 (F) and SK-OV-3 (G) cells. $\mathrm{n}=3 ;{ }^{*}, \mathrm{p}<0.05$.
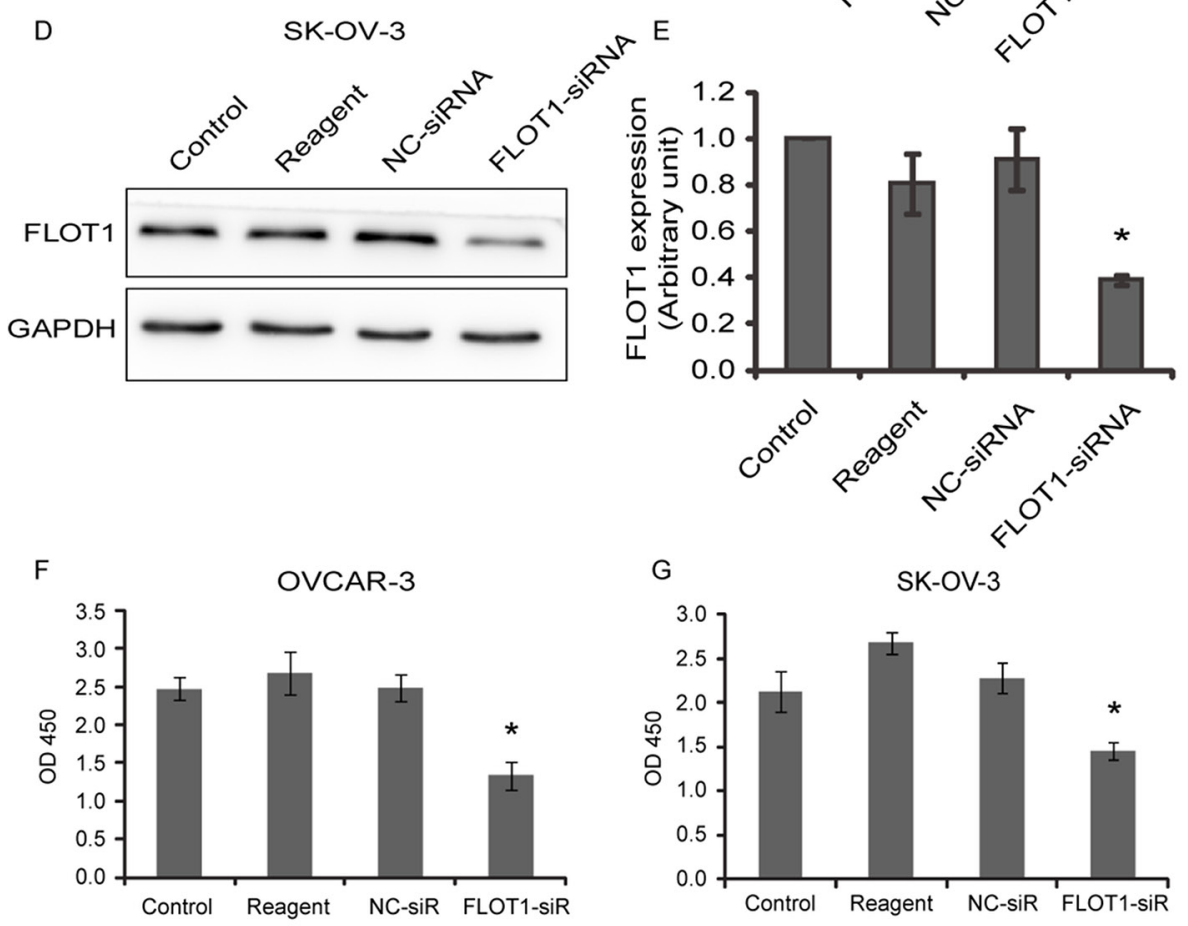

\section{Discussion}

Ovarian cancer is a heterogeneous group of neoplasms which includes serous, mucinous, endometrioid, clear cell, transitional and other cell sub-types. These differ in pathogenesis and molecular initiating events and therefore have different biological behavior and prognosis $[26,27]$. Recent studies have focused on the molecular biology and genetics of the different subtypes of ovarian cancer [28-30], and although the standard therapy for ovarian cancers is surgery followed by chemotherapy some sub-types such as mucinous and clear cell tumors have proved resistant to widely used chemotherapeutic agents [31,32].

FLOT1 expression is increased in various carcinomas and has been associated with tumorigenesis and progression by promoting tumor proliferation, invasiveness and 

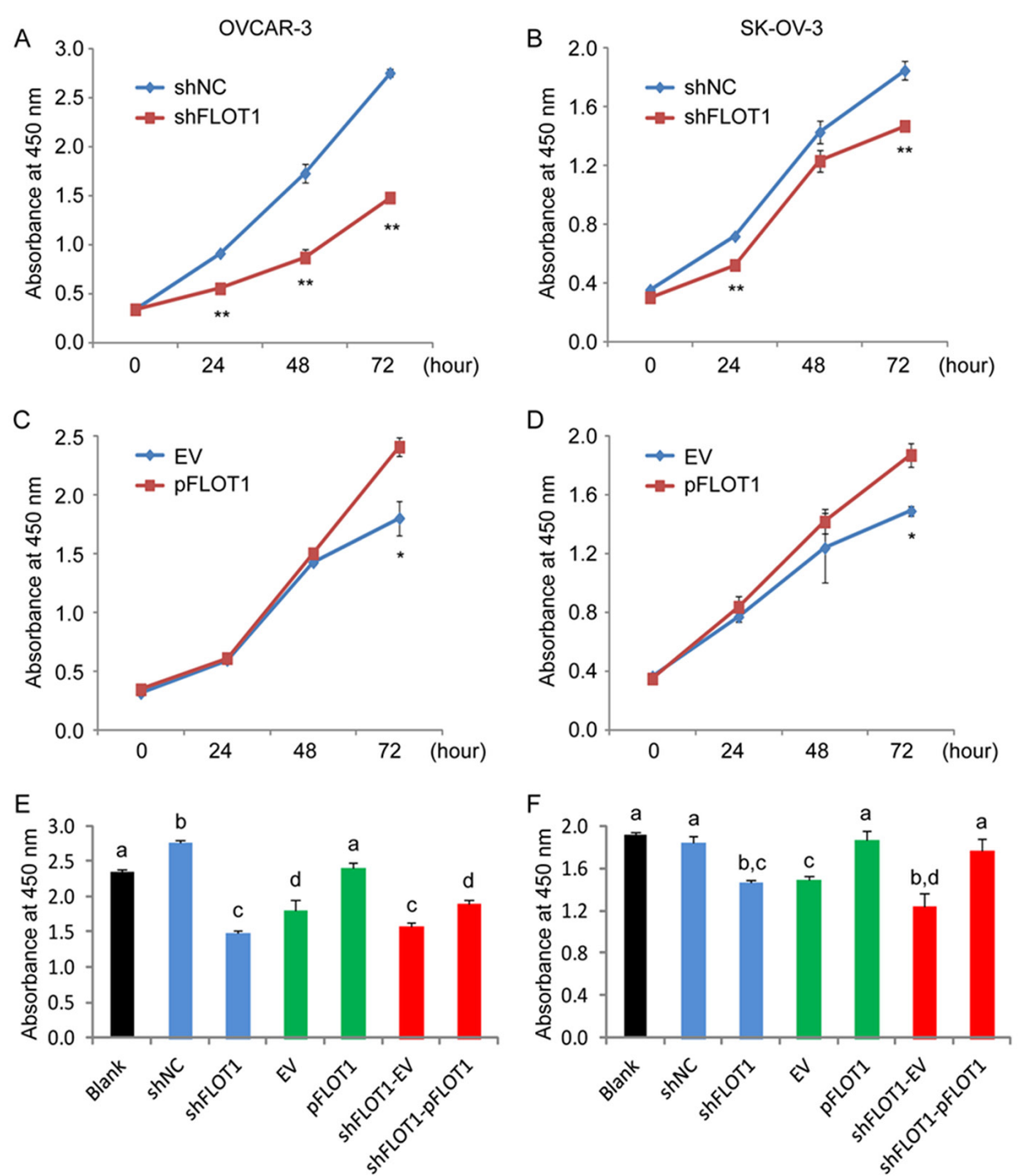

Figure 4. FLOT1 gain- and loss-of-function effect on cell proliferation in ovarian cancer cells. OVCAR-3 (A) and SK-OV-3 (B) cells were stably infected with FLOT1-shRNA or NC-shRNA lentivirus to generate the FLOT1-shRNA-expressing cells (shFLOT) and NC-shRNA-expressing cells (shNC). Cell proliferation was measured after culture for 24, 48 and 72 hours. OVCAR-3 (C) and SK-OV-3 (D) cells were transfected with FLOT1 plasmid DAN (pFLOT1) or empty vector (pEV). Cell proliferation was measured after transfection for 24,48 and 72 hours. The cell proliferation of OVCAR-3 (E) and SK-OV-3 (F) and their derived cells was measured after 72 hours culture. Blank, non-transfected cells; shNC, negative control-shRNA-expressing cells; shFLOT1, FLOT1-shRNA expressing cells; EV, empty vector transfecting cells; pFLOT1, FLOT1-plasmid transfecting cells; shFLOT1-EV, FLOT1shRNA-expressing cells transfected with EV; shFLOT1-pFLOT1, FLOT1-shRNA-expressing cells transfected with FLOT1 plasmid (pFLOT1). n=3; *, $\mathrm{p}<0.05 ;{ }^{\star *}, \mathrm{p}<0.01$. Data with different superscripts differed significantly.

metastasis via several signal pathways [33, 34]. We established that FLOT1 expression is higher in most malignant ovarian tumors than in benign tumor and normal ovarian tissue, and that it differed in ovarian tumor sub-types. Positive FLOT1 protein immunohistochemical staining was observed in different ovarian malignant tumors and these differences have not been reported in other cancers; perhaps because of ovarian cancer diversity. The differences in FLOT1 expression suggest that it could be a novel tissue marker for diagnosis and distinction between ovarian cancer sub-types, and it could also be a new therapeutic target for some OC's.
Previous studies found that FLOT1 expression is positively correlated with clinical variables, including tumor size, clinical stage, lymph node metastasis and pathologic grade $[10,12,14]$ that are not in line with our study findings. There was disparity with our finding that FLOT1 protein expression only correlated with pathological type and not with clinicopathological characteristics such as age, clinical stage, histological grade and lymph node metastasis. Because of the FLOT1 expression differences in OC sub-types, we excluded those with the smallest incidences and included only serous ovarian tumors in our analysis because this had significant 


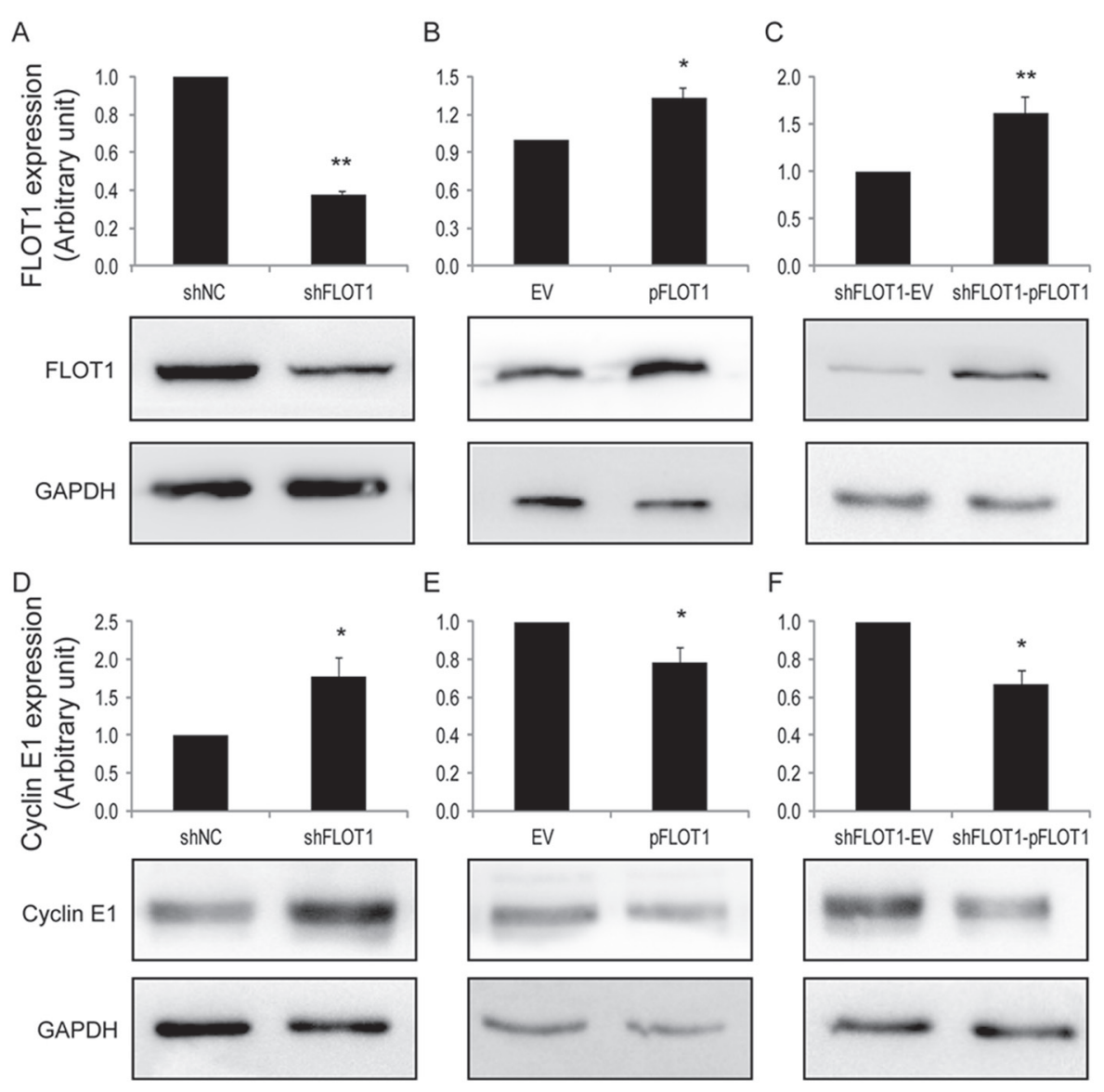

Figure 5. Expression of FLOT1 and cyclin E1 protein in SK-OV-3 cells detected by Western blot. A) Expression of FLOT1 in FLOT1-shRNA-expressing cells (shFLOT1) and negative control (NC)shRNA-expressing cells $(\mathrm{shNC})(\mathrm{n}=4)$. B) Expression of FLOT1 in cells transfected with FLOT1-plasmid (pFLOT1) and empty vector control plasmid (EV) $(n=3)$. C) Expression of FLOT1 in shFLOT1-expressing cells after pFLOT1 or EV transfection $(n=4)$. D) Expression of cyclin E1 in shFLOT1 and shNC cells $(n=6)$. E) $E x$ pression of cyclin $\mathrm{E} 1$ in cells transfected with pFLOT1 and EV (n=5). F) Expression of cyclin E1 in shFLOT1-expressing cells after pFLOT1 or EV transfection $(n=3)$. ${ }^{*}, \mathrm{p}<0.05 ;{ }^{* *}, \mathrm{p}<0.01$.
Figure 6. Cell cycle in ovarian cancer cells detected by flow cytometry. A) Images of flow cytometry on cell cycle after FLOT1siRNA and negative control siRNA (NCsiR) transfection. B) Histogram shows the percentage of cell population in each phase of $(A) . n=4 ;{ }^{*} p<0.05$.

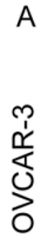

Blank
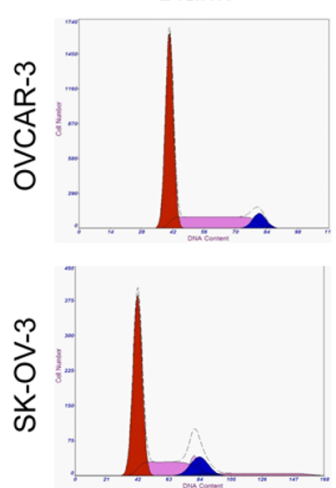

OVCAR-3

B

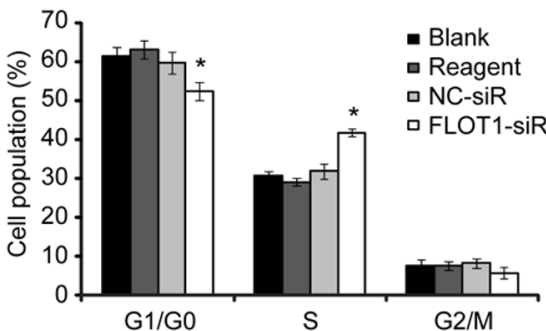

Reagent
NC-siR

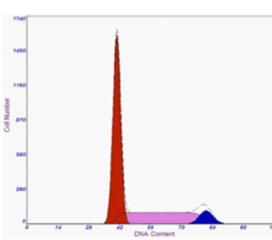

FLOT1-siR
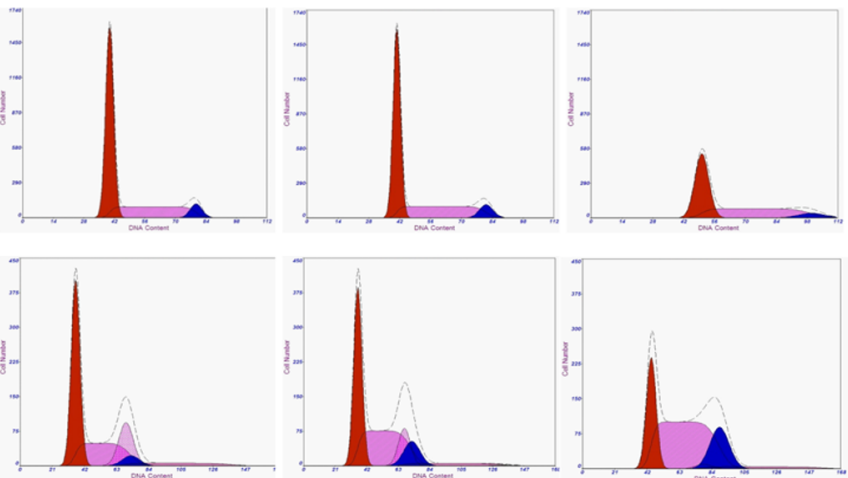

SK-OV-3

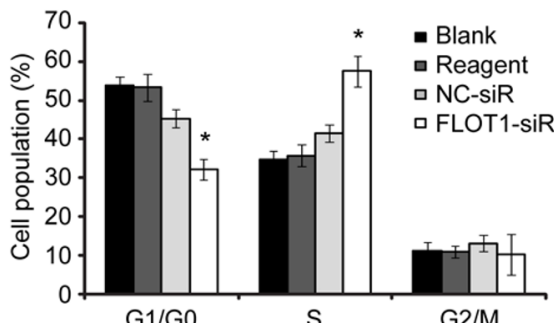


differences in malignant, borderline and benign tumors. Our results may also differ from previously reported data because of the obvious differences in OC and other cancer tumorigenesis.

FLOT1 is reported to be important in cell behavior [13] because its over-expression promotes, whereas down-regulation inhibits, cell proliferation. FLOT1 knockdown results in G1-S phase arrest of breast cancer cells with increased cell percentage in the G1/G0 phase and less in the S phase [13]. Silencing FLOT1 leads to increased p21 and p27 expression and decreased cyclin D and FOXO3a. Since FOXO3a is a transcriptional regulator for p21, p27 and cyclin D [35], FLOT1 may promote cell proliferation via the Akt/ FOXO3a pathway. Herein, FLOT1 expression knockdown led to significantly decreased proliferation in both OVCAR-3 and SK-OV-3 cells compared to controls, and while this is supported by previous studies, cell cycle assay results are inconsistent. Flow cytometry showed that cell cycle was arrested in the S phase of both OVCAR-3 and SK-OV-3 cells, with the percentage of cells in the $S$ phase significantly increased and those in the G1/G0 phase significantly decreased, thus indicating that FLOT1 suppression can arrest the OV cell cycle in the S phase while increasing cyclin E1 expression.

Previous studies demonstrate that cyclin E1 is essential for $S$ phase arrest in OC cells $[36,37]$. For example, Santamaría et al studied FLOT1influence on the cell cycle and cell proliferation, and found that both FLOT1 and PTVO1 enter the nucleus shortly before $S$ phase begins, remain in the nucleus throughout the $S$ phase and reach their peak 9 hours after serum stimulation [18]. FLOT1 protein nuclear localization is low during the G1/G0 phase, reaches its peak at the $S$ phase and then gradually declines. Those findings support the hypothesis that the reduced concentration of FLOT1 can promote $S$ phase entry when it is partly silenced, but it is insufficient to terminate the $S$ phase and enter the next phase. Cells depleted in FLOT1 protein accumulate in the S phase, resulting in a decreased percentage of cells in other phases. Further, if FLOT1 is sufficiently reduced, the S phase cannot be initiated and G1/G0 phase arrest therefore occurs.

This hypothesis also explains the differences in our results and Lin et al's., [13] but further study is needed to provide concrete evidence. Gomez et al's study of FLOT1 and the cell cycle found that FLOT1 knockdown causes down-regulation in Aurora B [38], a protein required for mitosis and a regulator of the mitotic checkpoint [39]. FLOT1 knockdown induced aberrant mitotic events and inhibited cell proliferation. Furthermore, the alteration in cell cycle regulators such as cyclin-dependent kinases (CDKs) and their inhibitors (p21 and p27) can also effect OC cell cycle arrest [40].

In conclusion, FLOT1 protein was over-expressed in several types of malignant ovarian tumors and its expression differed in the tested sub-types. Silencing FLOT1 through siRNA led to significant inhibition of OVCAR-3 and SK-OV-3 cell proliferation with cell cycle arrest at the S phase. While the effects of this over-expression are clear, the mechanisms of FLOT1 involvement in tumorigenesis and ovarian cancer progression require further study.

Acknowledgements: This work was supported by grants from the National Natural Science Foundation of China (81272880), Science and Technology Commission of Shanghai Municipality (124119b1300), Natural Science Foundation of Shanghai (17ZR1404100) and the Shanghai Municipal Commission of Health and Family Planning (201640287) to G. Xu, and the Jinshan District Commission of Health and Family Planning (JSKJ-KTMS-2014-08) to J. Li.

\section{References}

[1] TORRE LA, BRAY F, SIEGEL RL, FERLAY J, LORTETTIEULENT J et al. Global cancer statistics, 2012. CA Cancer J Clin 2015; 65: 87-108. https://doi.org/10.3322/caac.21262

[2] SMITH RA, ANDREWS K, BROOKS D, DESANTIS CE, FEDEWA SA et al. Cancer screening in the United States, 2016: A review of current American Cancer Society guidelines and current issues in cancer screening. CA Cancer J Clin 2016; 66: 96-114. https://doi.org/10.3322/caac.21336

[3] MILLER KD, SIEGEL RL, LIN CC, MARIOTTO AB, KRAMER JL et al. Cancer treatment and survivorship statistics, 2016. CA Cancer J Clin 2016; 66: 271-289. https://doi. org/10.3322/caac. 21349

[4] BABUKE T, RUONALA M, MEISTER $M$, AMADDII M, GENZLER C et al. Hetero-oligomerization of reggie-1/flotillin-2 and reggie-2/flotillin-1 is required for their endocytosis. Cell Signal 2009; 21: 1287-1297. https://doi.org/10.1016/j. cellsig.2009.03.012

[5] CREMONA ML, MATTHIES HJ, PAU K, BOWTON E, SPEED N et al. Flotillin-1 is essential for PKC-triggered endocytosis and membrane microdomain localization of DAT. Nat Neurosci 2011; 14: 469-477. https://doi.org/10.1038/ nn. 2781

[6] DERMINE JF, DUCLOS S, GARIN J, ST-LOUIS F, REA S et al. Flotillin-1-enriched lipid raft domains accumulate on maturing phagosomes. J Biol Chem 2001; 276: 18507-18512. https://doi.org/10.1074/jbc.M101113200

[7] WALTON JR, FREY HA, VANDRE DD, KWIEK JJ, ISHIKAWA T et al. Expression of flotillins in the human placenta: potential implications for placental transcytosis. Histochem Cell Biol 2013; 139: 487-500. https://doi.org/10.1007/ s00418-012-1040-2

[8] TOMIYAMA A, UEKITA T, KAMATA R, SASAKI K, TAKITA $J$ et al. Flotillin-1 regulates oncogenic signaling in neuroblastoma cells by regulating ALK membrane association. Cancer Res 2014; 74: 3790-3801. https://doi. org/10.1158/0008-5472.CAN-14-0241

[9] AMADDII M, MEISTER M, BANNING A, TOMASOVIC A, MOOZ J et al. Flotillin-1/reggie-2 protein plays dual role in activation of receptor-tyrosine kinase/mitogen-activated protein kinase signaling. J Biol Chem 2012; 287: 7265-7278. https://doi.org/10.1074/jbc.M111.287599 
[10] LI H, WANG RM, LIU SG, ZHANG JP, LUO JY et al. Abnormal expression of FLOT1 correlates with tumor progression and poor survival in patients with non-small cell lung cancer. Tumour Biol 2014; 35: 3311-3315. https://doi.org/10.1007/ s13277-013-1434-3

[11] SONG L, GONG H, LIN C, WANG C, LIU L et al. Flotillin-1 promotes tumor necrosis factor-alpha receptor signaling and activation of NF-kappaB in esophageal squamous cell carcinoma cells. Gastroenterology 2012; 143: 995-1005 e1012. https://doi.org/10.1053/j.gastro.2012.06.033

[12] GUAN Y, SONG H, ZHANG G, AI X. Overexpression of flotillin-1 is involved in proliferation and recurrence of bladder transitional cell carcinoma. Oncol Rep 2014; 32: 748-754. https://doi.org/10.3892/or.2014.3221

[13] LIN C, WU Z, LIN X, YU C, SHI T et al. Knockdown of FLOT1 impairs cell proliferation and tumorigenicity in breast cancer through upregulation of FOXO3a. Clin Cancer Res 2011; 17: 3089-3099. https://doi.org/10.1158/1078-0432. CCR-10-3068

[14] ZHANG SH, WANG CJ, SHI L, LI XH, ZHOU J et al. High Expression of FLOT1 Is Associated with Progression and Poor Prognosis in Hepatocellular Carcinoma. PLoS One 2013; 8: e64709. https://doi.org/10.1371/journal. pone.0064709

[15] FOUNTZILAS E, KOTOULA V, ANGOURIDAKIS N, KARASMANIS I, WIRTZ RM et al. Identification and validation of a multigene predictor of recurrence in primary laryngeal cancer. PLoS One 2013; 8: e70429. https://doi. org/10.1371/journal.pone.0070429

[16] ZHANG Y, LI J, SONG Y, CHEN F, PEI Y et al. Flotillin-1 expression in human clear-cell renal cell carcinoma is associated with cancer progression and poor patient survival. Mol Med Rep 2014; 10: 860-866. https://doi.org/10.3892/ mmr.2014.2310

[17] LI L, LUO J, WANG B, WANG D, XIE X et al. Microrna-124 targets flotillin-1 to regulate proliferation and migration in breast cancer. Mol Cancer 2013; 12: 163. https://doi. org/10.1186/1476-4598-12-163

[18] SANTAMARIA A, CASTELLANOS E, GOMEZ V, BENEDIT P, RENAU-PIQUERAS J et al. PTOV1 enables the nuclear translocation and mitogenic activity of flotillin-1, a major protein of lipid rafts. Mol Cell Biol 2005; 25: 19001911. https://doi.org/10.1128/MCB.25.5.1900-1911.2005

[19] YANG FQ, ZHANG HM, CHEN SJ, YAN Y, ZHENG JH. MiR-506 is down-regulated in clear cell renal cell carcinoma and inhibits cell growth and metastasis via targeting FLOT1. PLoS One 2015; 10: e0120258. https://doi.org/10.1371/journal.pone.0120258

[20] LI H, ZHANG Y, CHEN SW, LI FJ, ZHUANG SM et al. Prognostic significance of Flotillin 1 expression in clinically N0 tongue squamous cell cancer. Int J Clin Exp Pathol 2014; 7: 996-1003.

[21] SOWTER HM, ASHWORTH A. BRCA1 and BRCA2 as ovarian cancer susceptibility genes. Carcinogenesis 2005; 26: 1651-1656. https://doi.org/10.1093/carcin/bgi136

[22] PRAT J, RIBE A, GALLARDO A. Hereditary ovarian cancer. Hum Pathol 2005; 36: 861-870. https://doi.org/10.1016/j. humpath.2005.06.006
[23] WANG X, GUI L, ZHANG Y, ZHANG J, SHI J et al. Cystatin $\mathrm{B}$ is a progression marker of human epithelial ovarian tumors mediated by the TGF-beta signaling pathway. Int $\mathrm{J}$ Oncol 2014; 44: 1099-1106. https://doi.org/10.3892/ijo.2014.2261

[24] SUN W, GUI L, ZUO X, ZHANG L, ZHOU D et al. Human epithelial-type ovarian tumour marker beta-2-microglobulin is regulated by the TGF-beta signaling pathway. J Transl Med 2016; 14: 75. https://doi.org/10.1186/s12967-016-0832-x

[25] ZHOU D, ZHANG L, SUN W, GUAN W, LIN Q et al. Cytidine monophosphate kinase is inhibited by the TGF-beta signalling pathway through the upregulation of miR-130b3 p in human epithelial ovarian cancer. Cell Signal 2017; 35: 197-207. https://doi.org/10.1016/j.cellsig.2017.04.009

[26] MCCLUGGAGE WG. Morphological subtypes of ovarian carcinoma: a review with emphasis on new developments and pathogenesis. Pathology 2011; 43: 420-432. https://doi. org/10.1097/PAT.0b013e328348a6e7

[27] GILKS CB. Subclassification of ovarian surface epithelial tumors based on correlation of histologic and molecular pathologic data. Int J Gynecol Pathol 2004; 23: 200-205.

[28] MCCLUGGAGE WG. My approach to and thoughts on the typing of ovarian carcinomas. J Clin Pathol 2008; 61: 152163. https://doi.org/10.1136/jcp.2007.049478

[29] SHIH IM, KURMAN RJ. Ovarian tumorigenesis: a proposed model based on morphological and molecular genetic analysis. Am J Pathol 2004; 164: 1511-1518.

[30] SOSLOW RA. Histologic subtypes of ovarian carcinoma: an overview. Int J Gynecol Pathol 2008; 27: 161-174. https://doi. org/10.1097/PGP.0b013e31815ea812

[31] LEE S, GARNER EI, WELCH WR, BERKOWITZ RS, MOK SC. Over-expression of hypoxia-inducible factor 1 alpha in ovarian clear cell carcinoma. Gynecol Oncol 2007; 106: 311 317. https://doi.org/10.1016/j.ygyno.2007.03.041

[32] CLOVEN NG, KYSHTOOBAYEVA A, BURGER RA, YU IR, FRUEHAUF JP. In vitro chemoresistance and biomarker profiles are unique for histologic subtypes of epithelial ovarian cancer. Gynecol Oncol 2004; 92: 160-166.

[33] LIANG Z, WANG X, XU X, XIE B, JI A et al. MicroRNA-608 inhibits proliferation of bladder cancer via $\mathrm{AKT} / \mathrm{FOXO} 3 \mathrm{a}$ signaling pathway. Mol Cancer 2017; 16: 96. https://doi. org/10.1186/s12943-017-0664-1

[34] JI H, GREENING DW, BARNES TW, LIM JW, TAURO BJ et al. Proteome profiling of exosomes derived from human primary and metastatic colorectal cancer cells reveal differential expression of key metastatic factors and signal transduction components. Proteomics 2013; 13: 1672-1686. https://doi.org/10.1002/pmic.201200562

[35] HUANG H, TINDALL DJ. Dynamic FoxO transcription factors. J Cell Sci 2007; 120: 2479-2487. https://doi. org/10.1242/jcs.001222

[36] TYAGI A, SINGH RP, AGARWAL C, SIRIWARDANA S, SCLAFANI RA et al. Resveratrol causes Cdc2-tyr15 phosphorylation via ATM/ATR-Chk1/2-Cdc25C pathway as a central mechanism for $\mathrm{S}$ phase arrest in human ovarian carcinoma Ovcar-3 cells. Carcinogenesis 2005; 26: 1978-1987. https://doi.org/10.1093/carcin/bgi165 
[37] KANG K, NHO CW, KIM ND, SONG DG, PARK YG et al. Daurinol, a catalytic inhibitor of topoisomerase IIalpha, suppresses SNU-840 ovarian cancer cell proliferation through cell cycle arrest in S phase. Int J Oncol 2014; 45: 558-566. https://doi.org/10.3892/ijo.2014.2442

[38] GOMEZ V, SESE M, SANTAMARIA A, MARTINEZ JD, CASTELLANOS E et al. Regulation of aurora B kinase by the lipid raft protein flotillin-1. J Biol Chem 2010; 285: 2068320690. https://doi.org/10.1074/jbc.M110.130591
[39] MURATA-HORI M, WANG YL. The kinase activity of aurora $\mathrm{B}$ is required for kinetochore-microtubule interactions during mitosis. Curr Biol 2002; 12: 894-899.

[40] NAM EJ, KIM YT. Alteration of cell-cycle regulation in epithelial ovarian cancer. Int J Gynecol Cancer 2008; 18: 11691182. https://doi.org/10.1111/j.1525-1438.2008.01191.x 